\title{
Prime-boost vaccination with plasmid DNA followed by recombinant vaccinia virus expressing BgGARP induced a partial protective immunity to inhibit Babesia gibsoni proliferation in dogs
}

\author{
Shinuo Ca0 ${ }^{1 * *}$, Ahmed Abdelmoniem Mousa ${ }^{1 * *}$, Gabriel Oluga Aboge ${ }^{1,2}$, \\ Ketsarin Kamyingkird ${ }^{1}$, Mo Zhou' ${ }^{1}$, Paul Franck Adjou Moumouni', Mohamad Alaa Terkawi', \\ Tatsunori Masatani' ${ }^{1}$, Yoshifumi Nishikawa ${ }^{1}$, Hiroshi Suzuki ${ }^{1}$, Shinya Fukumoto ${ }^{1}$ and Xuenan Xuan ${ }^{1 *}$ \\ ${ }^{1}$ National Research Center for Protozoan Diseases, Obihiro University of Agriculture and Veterinary Medicine, Inada-cho, Obihiro, \\ Hokkaido 080-8555, Japan; ${ }^{2}$ Department of Public Health, Pharmacology and Toxicology, University of Nairobi, P.O. BOX 29053-00625 \\ Kangemi, Nairobi, Kenya
}

\begin{abstract}
A heterologous prime-boost vaccination regime with DNA and recombinant vaccinia virus (rvv) vectors expressing relevant antigens has been shown to induce effective immune responses against several infectious pathogens. In this study, we describe the effectiveness of the prime-boost strategy by immunizing dogs with a recombinant plasmid followed by vaccinia virus, both of which expressed the glutamic acid-rich protein (BgGARP) of Babesia gibsoni. The dogs immunized with the prime-boost regime developed a significantly high level of specific antibodies against BgGARP when compared with the control groups. The antibody level was strongly increased after a booster immunization with a recombinant vaccinia virus. Two weeks after the booster immunization with a recombinant vaccinia virus expressing BgGARP, the dogs were challenged with B. gibsoni parasite. The dogs immunized with the prime-boost regime showed partial protection, manifested as a significantly low level of parasitemia. These results indicated that this type of DNA/rvv prime-boost immunization approach may have use against $B$. gibsoni infection in dogs.
\end{abstract}

\section{Keywords}

Babesia gibsoni, DNA vaccine, vaccinia virus, BgGARP

Babesia gibsoni is a tick-transmitted apicomplexan protozoan parasite that causes piroplasmosis in dogs. The infection is endemic in many regions of Asia, Africa, Europe, and the Americas (Casapulla et al. 1998). The disease is typically characterized by remittent fever, progressive anemia, hemoglobinuria, splenomegaly, hepatomegaly, and sometimes death (Birkenheuer et al. 2005). Recently, the disease is frequently present in dogs and has become a serious clinical problem (Suarez et al. 2001). Therefore, in order to control and allevi- ate the $B$. gibsoni infection in dogs, an effective vaccine is seen as a necessary step of an integrated preventive strategy. A previous study has indicated that a prime-boost immunization regime with a DNA plasmid and a recombinant vaccinia virus (rvv), both of which expressed a same antigen, could induce complete protection against Plasmodium sporozoite challenge using a mouse malaria model (Kumar et al. 2002). In our previous study, we identified a glutamic acid-rich protein (BgGARP) gene expressed on B. gibsoni merozoites (unpub- 
lished data). Although, the function has not been characterized, we demonstrated that the BgGARP was recognized as an immunodominant antigen by the host immune system in B. gibsoni-infected dogs. In this context, we demonstrated the immunogenicity of heterologous immunization with priming DNA-boosting recombinant vaccinia virus, both carrying the BgGARP gene, for inducing protection against $B$. gibsoni infection in dogs.

The entire gene encoding BgGARP was amplified from the $B$. gibsoni cDNA library by polymerase chain reaction (PCR). The PCR product was cloned into EcoRI restriction enzyme site under the control of the cytomegalovirus early enhancer/chicken beta actin (CAG) promoter of the eukaryotic expression vector pCAGGS (Niwa et al. 1991, Tokui et al. 1997), designated pCAGGS-BgGARP. The pCAGGS-BgGARP was amplified in a DH5 $\alpha$ strain of Escherichia coli, and the purification was performed using the QIAGEN Plasmid Mega Kit (Qiagen, Germany) according to the manufacturer's instructions. In addition, the entire BgGARP gene was cloned into the SalI site of the vaccinia virus transfer vector pAK8 (Yasuda, et al. 1990). The rabbit kidney cells (RK13) infected with the parent vaccinia virus LC16mO (mO) (Yasuda et al. 1990) strain were transfected with the pAK8-BgGARP using a lipofectine reagent (Gibco BRL, USA). The recombinant vaccinia virus expressing BgGARP (rvvBgGARP) was propagated in RK13 cells. The expression of the BgGARP in both of the recombinant plasmid and vaccinia virus was confirmed by using RK13 cells in vitro prior to an in vivo trial with dogs. RK13 cells were transfected with pCAGGS-BgGARP using a lipofectine reagent (Gibco BRL, USA) by the standard method. Then, the pCAGGS-BgGARP plasmid and rvvBgGARP were used for the further immunization in dogs.

Purebred female specific pathogen-free (SPF) beagle dogs (14-15 months; NihonNosan, Japan) were used in this study. The dog experiments in this article were conducted in accordance with the Guiding Principles for the Care and Use of Research Animals promulgated by Obihiro University of Agriculture and Veterinary Medicine. The dogs were divided into three groups and then immunized ( $\mathrm{n}=3$ /each group). The BgGARP-immunized group was vaccinated with pCAGGSBgGARP followed by rvvBgGARP. The control immunized group was vaccinated with pCAGGS empty plasmid followed by the parent vaccinia virus $\mathrm{mO}$ strain. The remaining group received no immunization treatment. The immunization regime used DNA priming three times and a vaccinia virus boosting once because this immunization schedule showed most effective result for the induction of a strong immune response against malaria infection than other vaccination regimes in humans (Dunachie and Hill, 2003). For the plasmid DNA immunization, dogs were injected intramuscularly (IM) in the quadriceps muscle by a $1 \mathrm{ml}$ syringe with a $21 \mathrm{G}$ needle. Each single dose consisted of $200 \mu \mathrm{g}$ of DNA dissolved in $1 \mathrm{ml}$ of phosphate buffered saline (PBS) containing 25\% (w/v) sucrose. The dogs were immunized three times at two-week intervals. Two weeks after the final DNA immunization, the dogs were boosted with $5 \times 10^{8} \mathrm{PFU}$ of the recombinant vaccinia virus rvvBgGARP or mO intravenously (IV). After the DNA or recombinant vaccinia virus immunization, dogs were examined every day for 14 days by a veterinarian and no side effect were observed.

To further characterize the immune response stimulated by the prime-boost strategy, levels of the IgG subtypes were assessed using the rBgGARP after booster immunization. The sera of dogs for antibody detection were collected from the vein of lower extremity. The levels of BgGARP specific total immunoglobulin $\mathrm{G}(\mathrm{IgG}), \operatorname{IgG} 1$, and $\operatorname{IgG} 2$ in dog sera were measured by enzyme-linked immunosorbent assay (ELISA) (Fukumoto et al. 2007). Furthermore, to determine whether immune responses generated against BgGARP were sufficient for providing protection against $B$. gibsoni infection, a purebred female SPF Beagle dog was used for the donor of the B. gibsoni NRCPD strain parasites for the challenge infection (Ishimine et al. 1978, Fukumoto et al. 2000). Then, the fresh infected red blood cells (RBCs) were collected at acute phase of the experimentally infected donor dog (approximately 13\% parasitemia, 14 days post-infection), and washed with PBS three times by centrifugation, and then adjusted to $2 \times 10^{8} \mathrm{ml}^{-1}$ in PBS. The infected RBCs were used for the challenge within in $1 \mathrm{~h}$ from the blood collection. Two weeks after the booster immunization, dogs were injected i.v. with $2 \times 10^{8}$ of $B$. gibsoni-infected RBCs in the intermediate cephalic vein. Parasitemia in peripheral blood and the packed cell volume (PCV) were monitored at 1 day intervals.

To examine whether the pCAGGS-BgGARP plasmid correctly expresses BgGARP, we transfected the plasmid to RK13 cells and detected it by immunoflurescence antibody test (IFAT). In the IFAT analysis, the mouse anti-rBgGARP antibody specifically reacted to the RK13 cells transfected with the pCAGGS-BgGARP plasmid 2 days post-transfection (Fig. 1A), but the transfected cells were not recognized by the non-immunized mice sera included as a negative control (Fig. 1B). In addition, RK13 cells were infected at $5 \mathrm{PFU} /$ cell with a rvvBgGARP. After incubating for 2 days, cells infected with rvvBgGARP were analyzed by IFAT using mouse antirBgGARP antibody or non-immunized mice sera. The specific fluorescence was observed in the cells infected with rvvBgGARP (Fig. 2C). In contrast, the infected cells were not recognized by the non-immunized mice sera (Fig. 1D). The results indicated that the BgGARP was correctly expressed in the RK13 cells.

In addition, the serological response (IgG, $\operatorname{IgG} 1$, and $\operatorname{IgG} 2$ antibodies) was evaluated by ELISA using rBgGARP as specific antigen and serum samples from all the dogs obtained at different time of the experiment (Fig. 2). The total IgG responses against BgGARP of the BgGARP immunized dogs were under detectable level by each DNA immunization. However, two weeks after the booster immunization with rvvBgGARP, the antibody response was significantly increased (Fig. 2A, day -14 vs. day 0). Although there is no 

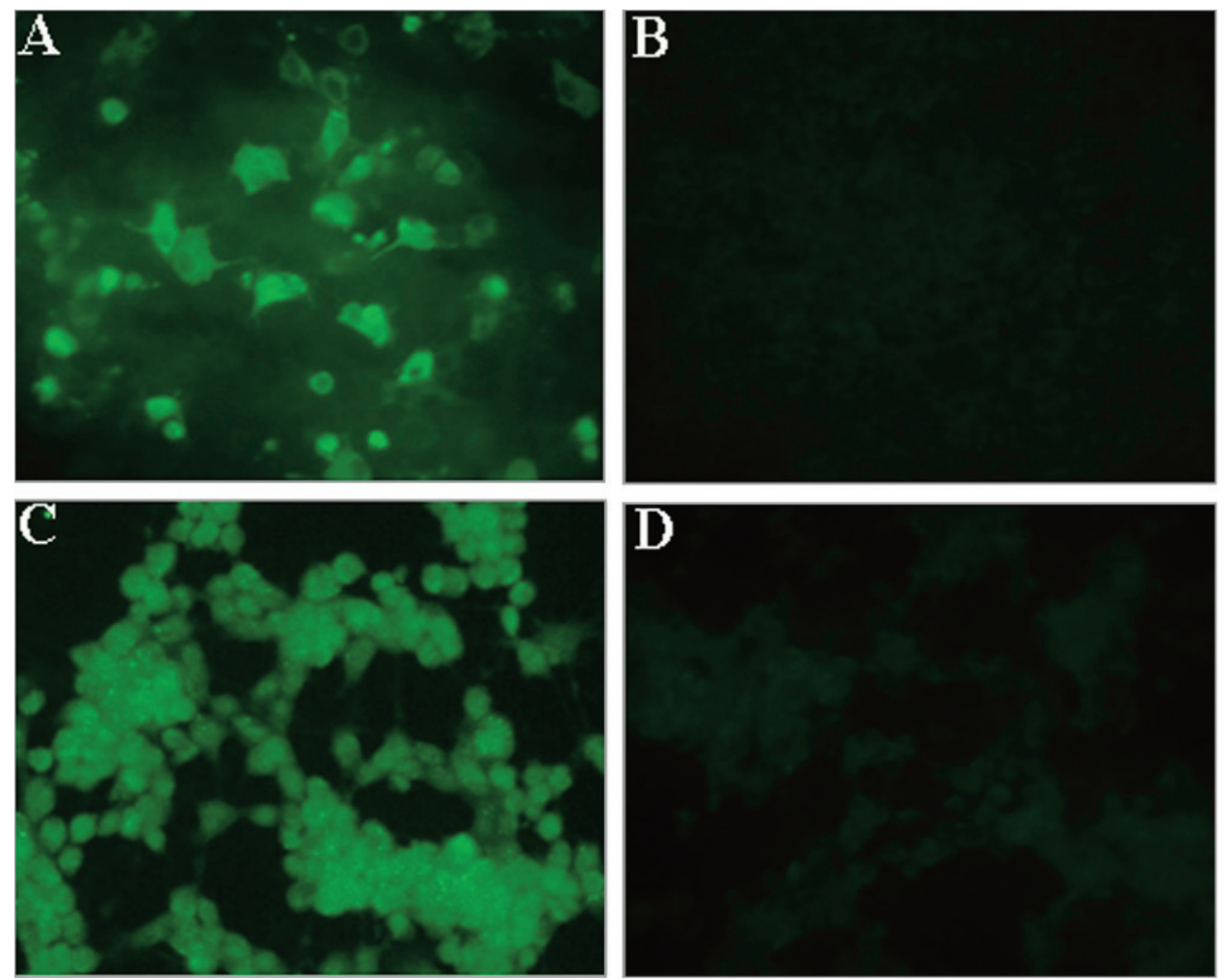

Fig. 1. Detection of BgGARP expression in RK13 cells. A. IFAT analysis of the BgGARP expressed in RK13 cells transfected with pCAGGSBgGARP. The cells were stained with mouse anti-BgGARP antibody. B. IFAT analysis of the BgGARP expressed in RK13 cells transfected with pCAGGS-BgGARP. The cells were stained with uninfected mouse antibody as a negative control. C. IFAT analysis of the BgGARP expressed in RK13 cells infected with rvvBgGARP. The cells were stained with mouse anti-BgGARP antibody. D. IFAT analysis of the BgGARP expressed in RK13 cells infected with rvvBgGARP. The cells were stained with uninfected mouse antibody as a negative control

correlation between total $\operatorname{IgG}$ level and the protective immunity, the antibody kinetics can be used as a marker for the vaccination. Furthermore, the IgG subclass against BgGARP was also analyzed. The IgG1 antibody response maintained a low level (Fig. 2B). The IgG1 response of the dogs immunized with BgGARP did not show significance compared to that of the control groups. In contrast, the IgG2 antibody was significantly increased after the booster immunization with rvvBgGARP (Fig. 2C, day -14 vs. day 0). When we compared IgG1 and IgG2, IgG2 was detected as major subclass all through the experimental period (Fig. $2 \mathrm{~B}$ and $\mathrm{C}$ ). The $\mathrm{IgG} 2$ response of the BgGARP group showed a significantly higher level (days 0 to 16, and 36) when compared to those of the control groups (Fig. 2C).

To evaluate the immunoprotective potential of the primeboost strategy against $B$. gibsoni infection, the dogs were inoculated with the parasites on day 0 and parasitemia was monitored by Giemsa-stained blood smears (Fig. 3). The parasitemia was significantly inhibited (day 18 to 22) in the dog group immunized with BgGARP when compared to both of the control groups. There was no significant difference in the two control groups. At the peak of parasitemia, the ratio of the inhibitory effect was $70.8 \%$ compared to that of the immunized control group and $73.8 \%$ compared to that of the non- immunized group. Despite the fact that the dogs immunized with BgGARP showed significantly low level of parasitemia, there was no significant difference in clinical symptoms manifested as severe anemia (Fig. 4). Thereafter, the immunized dogs showed little overall protection against effects such as anemia compared to the control groups. Although the mechanisms of the anemia is complicated and undefined, several reports have demonstrated that the parasitemia and the level of the anemia apparently lack of correlation. It may be associated the autoimmune hemolysis induced by pathogen infection (Daniel-Ribeiro et al. 2000). Therefore, the pathogenesis of $B$. gibsoni infection will be required to explore in the further study.

In conclusion, this study showed that dog vaccination priming with plasmid pCAGGS-BgGARP followed by a booster with rvvBgGARP triggered a high antibody response, leading to a significantly inhibition of $B$. gibsoni proliferation in dogs. The results demonstrate that the immunogenicity and efficacy of a heterologous prime-boost immunization with priming DNA followed by recombinant vaccinia virus boost strategy provided protective immunity against $B$. gibsoni infection. Therefore, the prime-boost regime expressing BgGARP may be potentially useful in the development of an effective vaccine controlling canine $B$. gibsoni infection. 


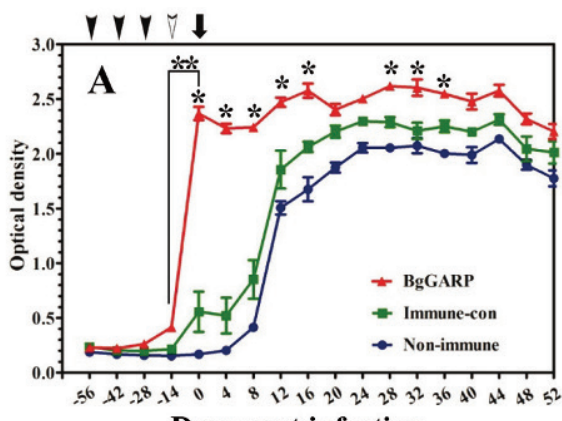

Days post infection

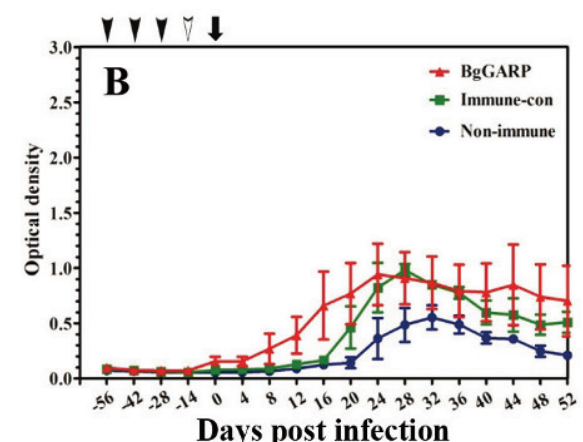

Days post infection

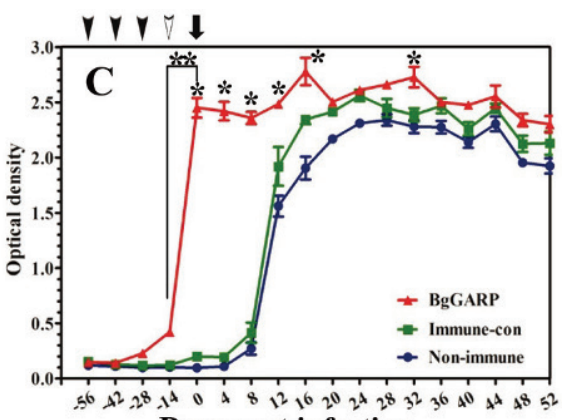

Days post infection

Fig. 2. Determination of the antibody response against BgGARP of dogs immunized with BgGARP. Antibody response against each immunization was monitored at two weeks after each immunization. A. Total IgG, B. IgG1 and C. IgG2. BgGARP, sera collected from dogs immunized with pCAGGS-BgGARP and rvvBgGARP; immune-con, sera collected from dogs immunized with control plasmid pCAGGS and $\mathrm{mO}$; non-immune, sera collected from non-immunized control dogs. The day at challenge infection of the parasites was designated as day 0 (solid arrow). The dogs were immunized with DNA at day $-56,-42$, and -28 (solid arrowhead), and immunized with vaccinia virus at day -14 (white arrow head). The asterisks $\left(^{*}\right)$ on the error bar show the significant difference $(P<0.05)$ between a dog group immunized with BgGARP and the control groups. The double asterisks $(* *)$ show the significant difference between day -14 and day 0 . The results are shown as the mean values, and the error bars represent the standard deviations

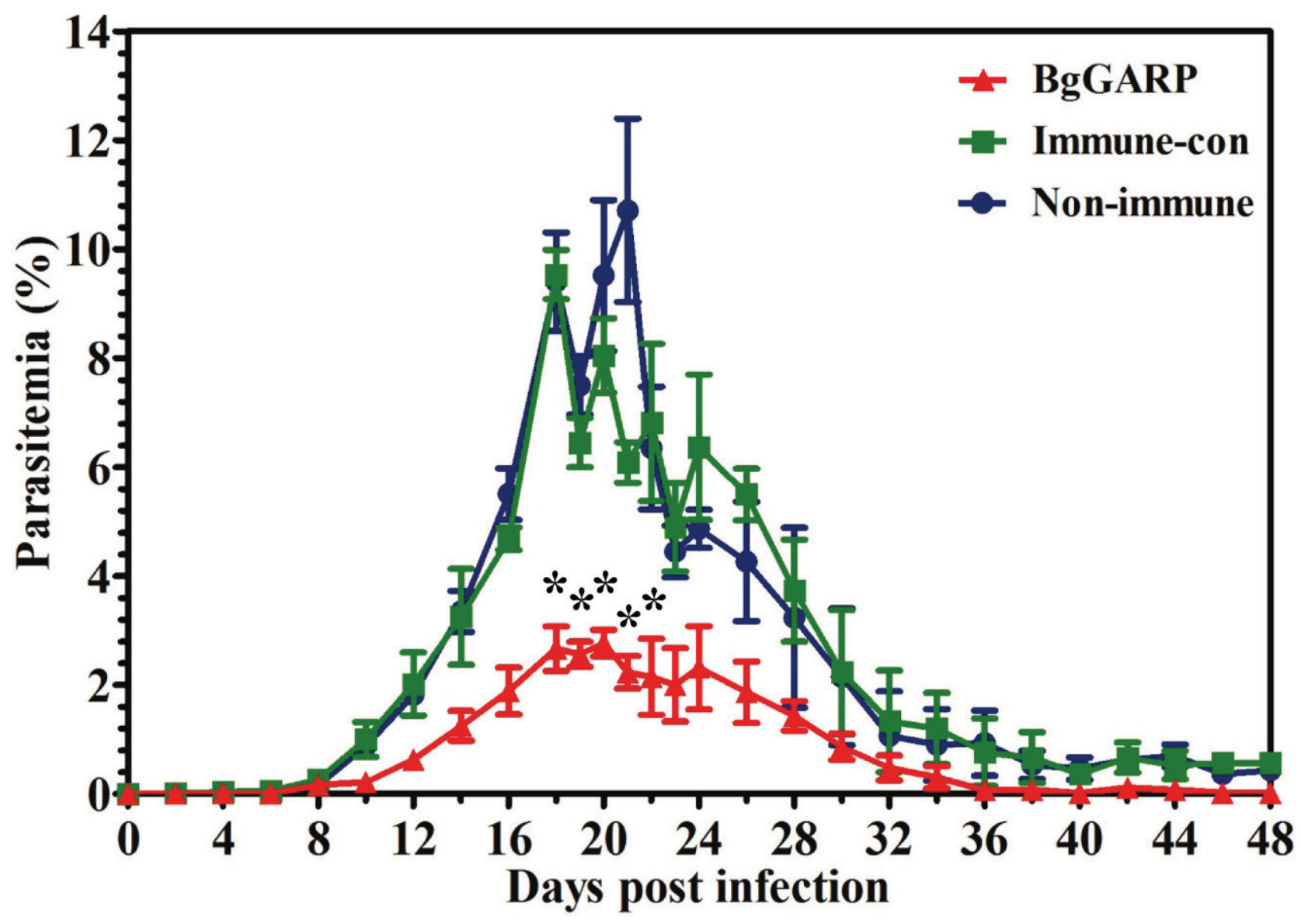

Fig. 3. Parasitemia of dogs after challenge infection with $B$. gibsoni-infected RBCs. BgGARP, dogs immunized with pCAGGS-BgGARP and rvvBgGARP; immune-con, dogs immunized with control plasmid pCAGGS and mO; non-immune, non-immunized control dogs. The results are shown as the mean values, and the error bars represent the standard deviations. The asterisks show the significant difference $(P<0.05)$ between a dog group immunized with BgGARP and the control groups 

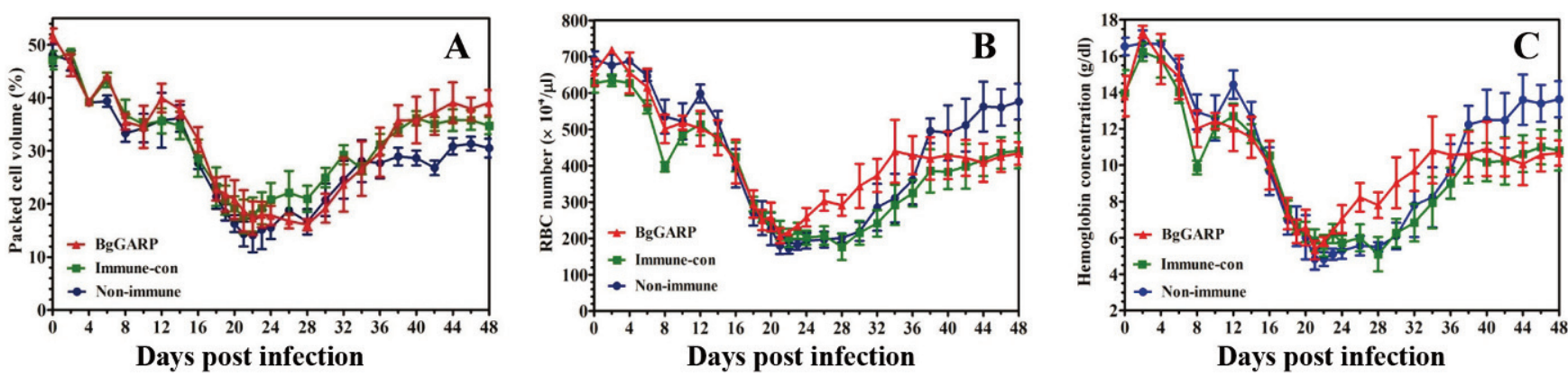

Fig. 4. Hematological parameters of the dogs after challenge infection with B. gibsoni-infected RBCs. A. Packed cell volume (PCV), B. RBC number, and C. hemoglobin concentration. BgGARP, dogs immunized with pCAGGS-BgGARP and rvvBgGARP; immune-con, dogs immunized with control plasmid pCAGGS and mO; non-immune, non-immunized control dogs. The results are shown as the mean values, and the error bars represent the standard deviations. Any significant difference was not observed between a dog group immunized with BgGARP and the control groups

Acknowledgements. This study was supported by a grant from the Global COE Program and Grants-in-Aid for Scientific Research, both from the Ministry of Education, Culture, Sports, Science, and Technology of Japan.

\section{References}

Birkenheuer A.J., Correa M.T., Levy M.G., Breitschwerdt E.B. 2005. Geographic distribution of babesiosis among dogs in the United States and association with dog bites: 150 cases (20002003). Journal of the American Veterinary Medical Association, 227, 942-947.

Casapulla R., Baldi L., Avallone V., Sannino R., Pazzanese L., Mizzoni V. 1998. Canine piroplasmosis due to Babesia gibsoni: clinical and morphological aspects. Veterinary Record, 142, 168-169.

Daniel-Ribeiro C.T., Zanini G. 2000. Autoimmunity and malaria: what are they doing together? Acta Tropica, 76, 205-221.

Dunachie S.J., Hill A.V. 2003. Prime-boost strategies for malaria vaccine development. Journal of Experimental Biology, 206, 3771-3779.

Fukumoto S., Tamaki Y., Okamura M., Bannai H., Yokoyama N., Suzuki, T., Igarashi I., Suzuki H., Xuan X. 2007. Primeboost immunization with DNA followed by a recombinant vaccinia virus expressing P50 induced protective immunity against Babesia gibsoni infection in dogs. Vaccine, 25, 1334-1341.

(Accepted: October 10, 2013)
Fukumoto S., Xuan X., Igarashi I., Zhang S., Mugisha J., Ogata T., Nagasawa H., Fujisaki K., Suzuki N., Mikami T. 2000. Morphological changes of Babesia gibsoni grown in canine red blood cell-substituted severe combined immune deficiency mice. Journal of Parasitology, 86, 956-958.

Ishimine T., Makimura S., Kitazawa S., Tamura S., Suzuki N. 1978. Pathophysiological findings on blood of Beagles experimentally infected with Babesia gibsoni. Japanese Journal of Tropical Medicine and Hygiene, 6, 15-26.

Kumar S., Epstein J.E., Richie T.L., Nkrumah F.K., Soisson L., Carucci D.J., Hoffman S.L. 2002. A multilateral effort to develop DNA vaccines against falciparum malaria. Trends in Parasitology, 18, 129-135.

Niwa H., Yamamura K., Miyazaki J. 1991. Efficient selection for high-expression transfectants with a novel eukaryotic vector. Gene, 108, 193-199.

Suarez M.L., Espino L., Goicoa A., Fidalgo L.E., Santamarina G. 2001. Fatal Babesia gibsoni infection in a dog from Spain. Veterinary Record, 148, 819-820.

Tokui M., Takei I., Tashiro F., Shimada A., Kasuga A., Ishii M., Ishii T., Takatsu K., Saruta T., Miyazaki J. 1997. Intramuscular injection of expression plasmid DNA is an effective means of long-term systemic delivery of interleukin-5. Biochemical and Biophysical Research Communications, 233, 527-531.

Yasuda A., Kimura-Kuroda J., Ogimoto M., Miyamoto M., Sata T., Sato T., Takamura C., Kurata T., Kojima A., Yasui K. 1990. Induction of protective immunity in animals vaccinated with recombinant vaccinia viruses that express PreM and Eglycoproteins of Japanese encephalitis virus. Journal of Virology, 64, 2788-2795. 